\title{
Clinical Features and Comparison of Kingella and Non-Kingella Endocarditis in Children, Israel
}

\begin{abstract}
Alexander Lowenthal, ${ }^{1}$ Hila Weisblum-Neuman, ${ }^{1}$ Einat Birk, Liat Ashkenazi-Hoffnung, Itzhak Levy, Haim Ben-Zvi, Gabriel Amir, Georgy Frenkel, Elchanan Bruckheimer, Havatzelet Yarden-Bilavsky, Dafna Marom, Eran Shostak, Elhanan Nahum, Tamir Dagan, Gabriel Chodick, Oded Scheuerman
\end{abstract}

\begin{abstract}
Kingella spp. have emerged as an important cause of invasive pediatric diseases. Data on Kingella infective endocarditis (KIE) in children are scarce. We compared the clinical features of pediatric KIE cases with those of Streptococcus species IE (StIE) and Staphylococcus aureus IE (SalE). A total of 60 patients were included in the study. Throughout the study period, a rise in incidence of KIE was noted. KIE patients were significantly younger than those with StIE and SalE, were predominately boys, and had higher temperature at admission, history of oral aphthae before IE diagnosis, and higher lymphocyte count $(p<0.05)$. Pediatric KIE exhibits unique features compared with StIE and SaIE. Therefore, in young healthy children $<36$ months of age, especially boys, with or without a congenital heart defect, with a recent history of oral aphthae, and experiencing signs and symptoms compatible with endocarditis, Kingella should be suspected as the causative pathogen.
\end{abstract}

Tnfective endocarditis (IE) is a rare but potentially life-threatening disease in children and has an incidence of $0.8-3.3$ cases $/ 1,000$ pediatric hospital admissions (1). Although early reports described IE exclusively in children whose hearts were structurally abnormal because of congenital heart disease or acquired rheumatic heart disease, this infection has

Author affiliations: Tel-Aviv University, Tel-Aviv, Israel

(A. Lowenthal, H. Weisblum-Neuman, E. Birk, L. Ashkenazi-

Hoffnung, I. Levy, H. Ben-Zvi, G. Amir, G. Frenkel,

E. Bruckheimer, H. Yarden-Bilavsky, D. Marom, E. Shostak,

E. Nahum, T. Dagan, G. Chodick, O. Scheuerman); Schneider

Children's Medical Center of Israel, Petach-Tikva, Israel

(A. Lowenthal, H. Weisblum-Neuman, E. Birk, L. Ashkenazi-

Hoffnung, I. Levy, G. Amir, G. Frenkel, E. Bruckheimer, H. Yarden-

Bilavsky, E. Shostak, E. Nahum, T. Dagan, O. Scheuerman);

Rabin Medical Center, Beilinson Hospital, Petach-Tikva (H. Ben-Zvi);

Tel Aviv Sourasky Medical Center, Tel Aviv (D. Marom)

DOI: https://doi.org/10.3201/eid2703.203022 more recently been reported in diverse groups of patients. In addition to children with congenital heart disease, other groups of children have emerged as being at high risk for IE, including children born prematurely; those with noncardiac congenital malformations, genetic syndromes, and malignancies; and, in particular, children with central venous catheters and those who have been treated by invasive procedures or intravenous medications (1-3).

The most common IE pathogens in children are gram-positive cocci, especially the a-hemolytic viridans group streptococci (e.g., Streptococcus sanguis, S. mitis group, and S. mutans), staphylococci, and enterococci. In patients with IE who are $>1$ year of age, the viridans group streptococci are the most commonly isolated organisms. Staphylococcus aureus is the second most common cause of IE in children but the most common cause of acute bacterial endocarditis (2). The HACEK group (Haemophilus parainfluenzae, H. aphrophilus, H. paraphrophilus, Aggregatibacter actinomycetemcomitans, Cardiobacterium hominis, Eikenella spp., and Kingella kingae) is a rare cause of IE, accounting for $\approx 1.4 \%$ of all cases of endocarditis $(2,4)$.

Kingella spp. are carried asymptomatically in the oropharynx and disseminate through close interpersonal contact. These gram-negative bacteria (especially K. kingae) are commonly the etiology of pediatric bacteremia and the leading cause of osteomyelitis and septic arthritis in children 6-36 months of age (5). Invasive K. kingae disease usually affects previously healthy children $<4$ years of age, whereas older children and adults frequently have predisposing conditions (6).

Kingella IE (KIE) is estimated to account for $0 \%-$ $6 \%$ of all IE cases in the general population (7-10). Similar numbers have been described in the pediatric population in a few published reports. Kingella

${ }^{1}$ These authors contributed equally to this article. 
appears to cause an even higher number of endocardial infections in children and was the etiologic agent of $4(7.8 \%)$ of 51 episodes in a tertiary-care pediatric hospital in Israel (3) and of $6(7.1 \%)$ of 85 cases among New Zealand children (11). However, lower rates of KIE have also been reported; a recent study of 53 cases of IE in Belgium described no cases of KIE (12). Serious cardiovascular and central nervous system complications and a need for emergent cardiac surgery for life-threatening complications that do not respond to conservative medical treatment have been described in the pediatric population (13). Kingella spp. as a causative pathogen of endocarditis has been poorly studied, and the number of studies regarding the pediatric population is limited $(3,11,14-20)$. Therefore, we examined the characteristics of pediatric KIE case-patients to compare these cases with IE cases caused by other common pathogens.

\section{Methods}

We retrospectively reviewed all files of children with IE admitted to Schneider Children's Medical Center of Israel (Petach-Tikva, Israel) during 1994-2019. We included children $\leq 18$ years of age with history and physical findings consistent with possible or definite diagnosis of IE according to the Duke criteria (21). Culture-negative IE cases were excluded because some might represent undiagnosed KIE. We also excluded cases of endocarditis that were attributed to coagulase-negative staphylococci species and other rare enteric gram-negative bacteria, because these consist of only nosocomial cases or IE cases associated with foreign bodies or intravenous catheters, which are epidemiologically distinct from the general IE pediatric population. A pediatric cardiologist and a pediatric infectious diseases specialist reviewed all files. Cases were divided into 2 groups on the basis of bacterial etiology: KIE (K. kingae and K. dentrificans) and non-Kingella IE (non-KIE, including Streptococcus species and $S$. aureus).

Each isolate was identified by using the VITEK 2 system (bioMérieux, https://www.biomerieux. com) or MALDI Biotyper System (Bruker, https:// www.bruker.com), in accordance with the manufacturers' instructions for bacteria identification. Antimicrobial-susceptibility profiles of the isolates were determined by the disk diffusion method (Oxoid, http://www.oxoid.com), Etest (bioMérieux), or VITEK 2 as needed and interpreted based on the Clinical and Laboratory Standards Institute criteria for other non-Enterobacteriaceae (22). Data retrieved from patients' charts included demographics, past medical history, clinical manifestations, laboratory findings, imaging studies, treatment, and outcome. The characteristics of KIE were compared with characteristics of Streptococcus species IE (StIE) and S. aureus IE (SaIE). The study was approved by the local institutional review board.

\section{Statistical Analysis}

To compare baseline correlates between case categories, we employed a $\chi^{2}$ test for categorical variables, analysis of variance test for parametric continuous data, and Kruskal-Wallis test for nonparametric continuous data. We calculated $p$ values for the post hoc comparison with Bonferroni correction for the number of comparisons. Statistical analyses were performed by using SPSS Statistics 23.0 software (IBM, https://www.ibm.com) and the tableone package (23) in $R$ version 3.6.1 ( $R$ Foundation for Statistical Computing, https:// www.r-project.org).

\section{Results}

\section{Study Population}

During the study period, IE was diagnosed in 114 admitted patients, yielding an incidence rate of 1.4 cases /1,000 admissions. A total of 60 patients with IE caused by Kingella species, Streptococcus species, or $S$. aureus were included in this study. In 19 patients $(14 \%$ of total IE admissions), the causative pathogen was Kingella species (K. kingae $[\mathrm{n}=18]$ and $K$. dentrificans $[n=1])$; in 25 patients $(19 \%)$, the causative pathogen was Streptococcus species (S. viridans [ $\mathrm{n}=17]$, S. pneumoniae $[\mathrm{n}=6]$, and S. pyogenes [ $\mathrm{n}=2]$ ); and in 16 patients $(12 \%)$, the causative pathogen was $S$. aureus.

\section{Baseline Characteristics}

The baseline characteristics of study participants with KIE and non-KIE are detailed in Table 1. Patients with KIE were significantly younger than those with nonKIE $(16 \pm$ SD 10.29 months vs. 91 + SD 74.11 months; $\mathrm{p}<0.001)$. Although the difference was not statistically significant, congenital heart disease was previously diagnosed in fewer patients with KIE than in patients with non-KIE ( $53 \%$ vs. $78 \%$; $p=0.09$ ). Based on queries regarding a previous heart murmur, far fewer patients with KIE had a history of a known murmur than those with non-KIE (37\% vs. $71 \%$; $=0.027)$. All KIE cases were community-acquired. No statistically significant differences were observed in previous noncardiac disease and previous interventions (surgery, cardiac catheterization, and dental procedures) between the groups. Median time (weeks) between prior cardiac catheterization to infection was shorter 
Kingella Endocarditis in Children, Israel

Table 1. Comparison of baseline demographics and characteristics of pediatric infective endocarditis case-patients by causative pathogen, Israel, 1994-2019*

\begin{tabular}{|c|c|c|c|}
\hline Characteristic & Kingella, $\mathrm{n}=19(32 \%)$ & Non-Kingella, $\mathrm{n}=41(68 \%)$ & $\mathrm{p}$ value \\
\hline Age, mo, mean (SD) & $16( \pm 10.29)$ & $91.4( \pm 74.11)$ & $<0.001$ \\
\hline \multicolumn{4}{|l|}{ Sex } \\
\hline $\mathrm{F}$ & $6(32)$ & $25(61)$ & 0.065 \\
\hline M & $13(68)$ & $16(39)$ & \\
\hline Congenital heart disease & $10(52)$ & $32(78.0)$ & 0.09 \\
\hline Known heart murmur & $7(37)$ & $29(71)$ & 0.027 \\
\hline Recent dental procedure & $0(0)^{\prime}$ & $6(15)$ & 0.195 \\
\hline Long-term CVL & $0(0)$ & $5(12)$ & 0.277 \\
\hline Recent catheterization & $4(21)$ & $6(15)$ & 0.804 \\
\hline Time from catheterization to infection, wk & $14.50(10-23) \ddagger$ & $6.00(2.9-14) \ddagger$ & 0.24 \\
\hline Community-acquired infection & $19(100)$ & $33(80)$ & 0.097 \\
\hline
\end{tabular}

*Values are no. (\%) except as indicated. CVL, central venous line.

†Recent catheterization or dental procedures defined as $\leq 6$ mo before diagnosis of infective endocarditis.

‡Median (interquartile range).

for the non-KIE group than the KIE group but was not statistically significant.

\section{Clinical, Laboratory, and Imaging Characteristics}

We compiled the clinical, laboratory, and imaging characteristics of case-patients with KIE compared with non-KIE case-patients (Table 2). Patients with KIE had significantly higher fever when first examined $\left(40^{\circ} \mathrm{C}\right.$ [range $39.45^{\circ} \mathrm{C}-40^{\circ} \mathrm{C}$ ] vs. $39^{\circ} \mathrm{C}$ [range $\left.\left.38.6^{\circ} \mathrm{C}-39.8^{\circ} \mathrm{C}\right] ; p=0.003\right)$. No difference was observed in duration of febrile disease before admission. Hepatosplenomegaly was more common among non-KIE patients. Approximately a quarter of KIE patients reported previous oral aphthae, significantly more than those in the non-HIE group ( 5 patients vs. 0 patients; $\mathrm{p}=0.003)$. No additional differences in clinical findings were noted.

The leukocyte count at admission differed significantly in lymphocyte counts: $4.27 \mathrm{~K}$ cells/mL (3.04) among KIE case-patients vs. $2.21 \mathrm{~K}$ cells/mL (1.81) in non-KIE case-patients $(p=0.002)$. Study groups approached significance $(p=0.055)$ in neutrophil-lymphocyte ratio; patients with KIE had the lowest ratio (4.7), whereas non-KIE patients had a higher ratio (10.7). No differences were observed in other parameters of the complete blood count or the level of inflammatory markers between the 2 groups. The number of positive cultures differed significantly between the 2 groups; most patients with KIE had 1-2 positive blood cultures, and none had $\geq 4$ positive cultures, compared with an average of 4 in the non-KIE group $(p<0.001)$. Days to blood-culture sterilization were fewer in the KIE group (2 days [2-3] vs. 3 days [2-5]; $p=0.017$ ). The chest radiography or echocardiography findings did not exhibit differential features between the 2 groups.

Duke criteria findings are listed in Table 2. Only $37 \%$ of those patients with KIE versus $98 \%$ in the nonKIE group $(\mathrm{p}<0.001)$ fulfilled the Duke major clinical criterion blood culture component. However, blood- culture positivity as a minor clinical criterion was far more prevalent in the KIE group than the non-KIE group (11 patients [58] vs. 1 patient [2]; $p<0.001$ ).

A post hoc comparison of the 3 pathogen groups (Table 3) showed that KIE differed significantly from the StIE group in a few parameters. Previous diagnosis of a heart murmur $(\mathrm{p}=0.027)$ and hepatosplenomegaly $(p<0.001)$ were less prevalent in patients with KIE. The absolute leukocyte count was significantly higher in the KIE group than in the StIE group $(p<0.005)$. KIE was significantly more likely to be community-acquired than SaIE $(p<0.012)$. Absolute neutrophil count was significantly lower in the KIE group than the SaIE group $(\mathrm{p}<0.001)$.

\section{Outcome}

Complications and mortality rates are shown in Table 4. No statistically significant differences were found between case-patients with KIE and those with nonKIE. Urgent surgery $\leq 10$ days after admission was more common in the KIE group but did not reach statistical significance. No deaths occurred in the KIE group, whereas the non-KIE group had an intrahospitalization death rate of $17 \%$.

\section{Discussion}

In this study we described the distinct features of pediatric KIE in a large cohort. We found that pediatric patients with KIE have similar characteristics, enabling the suspicion of Kingella as a causative pathogen when patients seek care. KIE is community-acquired and occurs in children (mean age 16 months) who are experiencing hyperpyrexia and have no history of previous structural heart disease. A quarter of patients in this study had a history of oral aphthae. This finding is consistent with previous studies indicating that Kingella are often carried in the oropharynx of toddlers and that oral aphthae are the port of entry resulting in bacteremia $(5,6)$. 
Relative lymphocytosis was found to be significantly more prevalent in the KIE group than the nonKIE group (4.27 K leukocytes/mL vs. $2.21 \mathrm{~K}$ leukocytes $/ \mathrm{mL}$ ). This finding is probably because of the younger age of KIE case-patients. Children with KIE had fewer positive blood cultures and shorter duration of positive cultures. When examining the Duke criteria, we found that a minority of KIE case-patients fulfilled microbiologic major criteria compared with non-KIE case-patients. Because the infection was community-acquired in all patients with KIE and only about half had structural heart disease, in most cases only 1 culture was drawn, probably because of the low level of suspicion. This practice might explain why culture positivity as a minor criterion was far more prevalent in the KIE group than the non-KIE group (57.9\% vs. $2.4 \%)$. We can therefore assume that, in most cases of KIE, the diagnosis was not clear at admission and that non-KIE pathogens require prolonged antimicrobial regimens for eradication.

A previous study in Israel suggests that the proportion of pediatric KIE cases in Israel is rising, from $4.2 \%$ of total IE cases during $1980-1991$ to $14 \%$ during 1994-2019 (24), consistent with the findings in our study. This high proportion of KIE has not been described previously in other countries $(15,25)$. A probable explanation is the improved detection of this fastidious bacterium, combined with the tertiary nature of our medical center. In addition, a higher prevalence of Kingella infection in Israel is a plausible explanation $(5,6)$.

Data characterizing the course of disease and fatal outcomes were not very helpful in differentiating between the groups, apart from deaths noted

Table 2. Comparison of clinical, laboratory, and imaging characteristics of pediatric infective endocarditis case-patients by causative pathogen, Israel, 1994-2019*

\begin{tabular}{|c|c|c|c|}
\hline Characteristic & Kingella, $\mathrm{n}=19(32 \%)$ & Non-Kingella, $\mathrm{n}=41(68 \%)$ & $p$ value \\
\hline Temperature, ${ }^{\circ} \mathrm{C}$, median (IQR) & $40(39.45-40)$ & $39(38.6-39.8)$ & 0.003 \\
\hline Fever duration before admission, $d$, median (IQR) & $7(4.50-14)$ & $6(3-14)$ & 0.43 \\
\hline Hepatosplenomegaly & $4(21)$ & $24(58)$ & 0.015 \\
\hline Oral aphthae & $5(26)$ & $0(0)$ & 0.003 \\
\hline Ocular findings & $1(5)$ & 8 (19) & 0.294 \\
\hline Systemic emboli & $7(37)$ & $14(34)$ & 1 \\
\hline Pulmonary emboli & $0(0.0)$ & $2(5)$ & 0.837 \\
\hline Seizures & $3(16)$ & $6(15)$ & 1 \\
\hline New onset murmur & $7(37)$ & $10(24)$ & 0.492 \\
\hline Conduction disturbance & $1(5)$ & $2(5)$ & 1 \\
\hline Microhematuria & $5(26)$ & $23(56)$ & 0.061 \\
\hline Leukocyte count $\mathrm{K} / \mathrm{mL}$, mean (SD) & $20.87( \pm 12.39)$ & $16.39( \pm 9.58)$ & 0.131 \\
\hline Neutrophils K/mL, mean (SD) & $12.98( \pm 8.49)$ & $12.43( \pm 8.67)$ & 0.821 \\
\hline Lymphocytes K/mL, mean (SD) & $4.27( \pm 3.04)$ & $2.21( \pm 1.81)$ & 0.002 \\
\hline NLR, mean (SD) & $4.7(6.71)$ & $10.7(11.3)$ & 0.055 \\
\hline Hemoglobin, mean (SD) & $10.00( \pm 1.36)$ & $10.39( \pm 2.27)$ & 0.489 \\
\hline Platelets $(100 \mathrm{~K} / \mathrm{mL})$, mean (SD) & $220.16( \pm 203.30)$ & $243.85( \pm 178.53)$ & 0.649 \\
\hline C-reactive protein, mean (SD) & $12.56( \pm 6.79)$ & $12.49( \pm 10.75)$ & 0.979 \\
\hline ESR, mean (SD) & $65.92( \pm 38.86)$ & $64.19( \pm 34.34)$ & 0.89 \\
\hline No. positive blood cultures, median (IQR) & $1(1-2)$ & $4(3-5)$ & $<0.001 \dagger$ \\
\hline Time to eradication, $\mathrm{d}$, median (IQR) & $2(2-3)$ & $3(2-5)$ & 0.017 \\
\hline \multicolumn{4}{|l|}{ Echocardiography } \\
\hline Mural thrombus & $1(5)$ & $2(5)$ & 1 \\
\hline Reduced ventricular function & $4(21)$ & $11(27)$ & 0.873 \\
\hline Vegetation & $12(63)$ & $20(49)$ & 0.447 \\
\hline Left-sided involvement $\ddagger$ & $12(80)$ & $15(65)$ & 0.297 \\
\hline \multicolumn{4}{|l|}{ Major Duke criteria } \\
\hline Culture & $7(37)$ & $40(98)$ & $<0.001$ \\
\hline Echocardiography & $16(84)$ & $23(56)$ & 0.067 \\
\hline \multicolumn{4}{|l|}{ Minor Duke criteria } \\
\hline Fever & $19(100)$ & $39(95)$ & 0.837 \\
\hline Congenital heart disease & $10(52)$ & $31(76)$ & 0.138 \\
\hline Vascular & $8(42)$ & $14(34)$ & 0.759 \\
\hline Immunologic & $0(0)$ & $9(22)$ & 0.068 \\
\hline Blood culture & $11(58)$ & $1(2)$ & $<0.001$ \\
\hline \multicolumn{4}{|l|}{ Summary } \\
\hline Definite & $14(74)$ & $30(73)$ & 0.768 \\
\hline Possible & $5(23)$ & $11(27)$ & 0.768 \\
\hline
\end{tabular}

*Values are no. (\%) except as indicated. All laboratory results are at admission apart from temperature, which we recorded as the highest in the $24 \mathrm{~h}$ before admission. ESR, erythrocyte sedimentation rate; IQR, interquartile range; NLR, neutrophil-lymphocyte ratio.

†By Mann-Whitney test.

†Percentage of left-sided involvement in patients with echocardiographic findings. 
Table 3. Comparison of the characteristics of case-patients with infective endocarditis by specific causative pathogen, Israel, 1994$2019^{*}$

\begin{tabular}{|c|c|c|c|c|}
\hline Characteristic & $\begin{array}{c}\text { Kingella species, } \mathrm{n}= \\
19 \\
\end{array}$ & $\begin{array}{c}\text { Streptococcus } \\
\text { species, } \mathrm{n}=25\end{array}$ & $\begin{array}{c}\text { Staphylococcus aureus } \\
\text { species, } \mathrm{n}=16\end{array}$ & $p$ value \\
\hline Age, mo, mean (SD) & $16( \pm 10.29)$ & $106.3( \pm 70.43)$ & $68( \pm 75.89)$ & $<0.001 \dagger$ \\
\hline \multicolumn{5}{|l|}{ Sex } \\
\hline $\mathrm{F}$ & $6(32)$ & $15(60)$ & $10(62)$ & 0.104 \\
\hline $\mathrm{M}$ & $13(68)$ & $10(40)$ & $6(38)$ & \\
\hline Congenital heart disease & $10(52)$ & $21(84)$ & $11(69)$ & 0.079 \\
\hline Known murmur & $7(37)$ & $19(76)$ & $10(62.5)$ & $0.031 \neq$ \\
\hline Recent surgery & $3(16)$ & $2(8)$ & $6(37)$ & $0.055^{\top}$ \\
\hline Recent dental procedure & 0 & $5(20)$ & $1(6.2)$ & 0.077 \\
\hline Community-acquired infection & $19(100.0)$ & $23(92.0)$ & $10(62.5)$ & $0.003 \S$ \\
\hline Temperature, ${ }^{\circ} \mathrm{C}$, median (IQR) & $40(39.45-40)$ & $39(39-39.6)$ & $39(38.4-40)$ & $0.013 \dagger$ \\
\hline Fever duration, d, median (IQR) & $7(4.5-14)$ & $7(2-21)$ & $5(3-7)$ & 0.514 \\
\hline Hepatosplenomegaly & $4(21)$ & $15(60)$ & $9(56)$ & $0.025 \ddagger$ \\
\hline Oral aphthae & $5(26)$ & 0 & 0 & $0.002 \dagger$ \\
\hline Musculoskeletal infection & $2(11.8)$ & 0 & 0 & 0.111 \\
\hline Microhematuria & $5(26.3)$ & $13(52.0)$ & $10(62.5)$ & 0.08 \\
\hline Leukocyte count, $\mathrm{K} / \mathrm{mL}$, mean (SD) & $20.87( \pm 12.39)$ & $12.68( \pm 4.77)$ & $22.18( \pm 12.23)$ & $0.005 \ddagger$ \\
\hline Neutrophils, $\mathrm{K} / \mathrm{mL}$, mean (SD) & $12.98( \pm 8.49)$ & $9.34( \pm 4.30)$ & $17.27( \pm 11.41)$ & $0.012 \S$ \\
\hline Lymphocytes, K/mL, mean (SD) & $4.27(+3.04)$ & $2.09( \pm 1.36)$ & $2.40( \pm 2.38)$ & $0.007 \dagger$ \\
\hline NLR, mean (SD) & $4.7(6.7)$ & $7.8(10.4)$ & $15.2(11.5)$ & $0.01 \ddagger$ \\
\hline C-reactive protein, mean (SD) & $12.56(+6.79)$ & $10.67( \pm 10.21)$ & $15.23( \pm 11.33)$ & 0.385 \\
\hline Reduced ventricular function & $4(21)$ & $4(16)$ & $7(44)$ & 0.12 \\
\hline Central nervous system involvement & $4(21)$ & $5(21)$ & $3(19)$ & 0.983 \\
\hline Death & 0 & $3(12.5)$ & $3(25)$ & 0.074 \\
\hline Culture positivity as major Duke criteria & $7(37)$ & $24(96)$ & $16(100)$ & $<0.001 \dagger$ \\
\hline Echocardiography as major Duke criteria & $16(84)$ & $13(52)$ & $10(62)$ & 0.083 \\
\hline Immunologic involvement as minor Duke criteria & 0 & $5(20)$ & $4(25)$ & 0.078 \\
\hline Culture as minor Duke criteria & $11(58)$ & $1(4)$ & 0 & $<0.001 \dagger$ \\
\hline Vegetation & $10(53)$ & $6(24)$ & $8(50)$ & 0.1 \\
\hline
\end{tabular}

only in the non-KIE cohort, which probably signify that most KIE case-patients were healthier before contracting IE. We discovered some similarities between the KIE group and the SaIE subgroup; however, larger numbers are needed to draw significant conclusions. The similar trends observed in these groups emphasize the high risk for major complications in KIE as observed in previous studies $(16,26)$. For reasons unknown, KIE causes devastating damage to the valve tissue in some cases but not others. This range of severity is probably explained by the different Kingella strains, which cause varying clinical syndromes (27). Unfortunately, K. kingae isolates of the patients in our study were not kept in our laboratory for further genotyping. A recent study postulated that a certain major virulence factor of $K$. kingae RtxA, a toxin that belongs to the RTX (repeats in toxin) group of secreted pore-forming toxins, is found in some $K$. kingae strains and causes cellular death by pore formation (28). Of note, S. aureusderived a-toxin, a pore-forming exotoxin, has also been implicated as a major cause of cardiac tissue damage in SaIE (29).

The limitations of our study include its retrospective data gathering and the relatively small cohort. We did not include cases of IE caused by coagulasenegative staphylococci and enteric gram-negative bacteria in the study. These pathogens cause only

\begin{tabular}{|c|c|c|c|}
\hline \multirow[b]{2}{*}{ Complication } & \multicolumn{2}{|c|}{ No. (\%) } & \multirow[b]{2}{*}{$p$ value } \\
\hline & Kingella IE & Non-Kingella IE & \\
\hline Surgical intervention & $8(42)$ & $15(37)$ & 0.958 \\
\hline Urgent surgical intervention† & $4(8)$ & $4(15)$ & 0.07 \\
\hline Congestive heart failure & $7(37)$ & 7 (17) & 0.192 \\
\hline Valvular impairment & $11(58)$ & $15(37)$ & 0.233 \\
\hline Central nervous system involvement & $4(21)$ & $8(20)^{\prime}$ & 1 \\
\hline Intrahospital death & $0(0)$ & $6(17)$ & 0.131 \\
\hline
\end{tabular}

trahospital death

${ }^{*} \mathrm{IE}$, infective endocarditis.

$t<10$ days after diagnosis. 
nosocomial and foreign body-associated endocarditis and occur in a distinct hospital-associated population. Including those bacteria would have biased this study by further emphasizing Kingella as a community-acquired cause of IE. We also excluded culture-negative cases of endocarditis because these could have included partially treated cases of Kingella endocarditis. An additional limitation is a selection bias of the population because our medical center is a tertiary-care center. Therefore, patients in whom endocarditis is diagnosed, patients with congenital heart disease, and patients with serious complications are referred to our center from other hospitals. Conversely, this bias is preserved in all 3 groups because most pediatric patients with IE are referred to a tertiary-care center.

In conclusion, this study shows that pediatric KIE has typical features compared with StIE and SaIE. Clinical cases of high fever in young healthy children (<36 months of age), especially boys, with or without congenital heart defects and with a recent history of oral aphthae should raise the suspicion for KIE.

\section{About the Author}

Dr. Lowenthal is a staff pediatric cardiologist at Schneider Children's Medical Center of Israel. His main research interests are fetal echocardiography, echocardiography, and acquired pediatric heart disease.

\section{References}

1. Day MD, Gauvreau K, Shulman S, Newburger JW. Characteristics of children hospitalized with infective endocarditis. Circulation. 2009;119:865-70. https:// doi.org/ 10.1161/CIRCULATIONAHA.108.798751

2. Ferrieri P, Gewitz MH, Gerber MA, Newburger JW, Dajani AS, Shulman ST, et al. Unique features of infective endocarditis in childhood. Pediatrics. 2002;109:931-43. https://doi.org/10.1542/peds.109.5.931

3. Marom D, Levy I, Gutwein O, Birk E, Ashkenazi S. Healthcare-associated versus community-associated infective endocarditis in children. Pediatr Infect Dis J. 2011;30:585-8. https://doi.org/10.1097/INF.0b013e31820f66c7

4. Chambers ST, Murdoch D, Morris A, Holland D, Pappas P, Almela M, et al.; International Collaboration on Endocarditis Prospective Cohort Study Investigators. HACEK infective endocarditis: characteristics and outcomes from a large, multi-national cohort. PLoS One. 2013;8:e63181. https:/ / doi.org/10.1371/journal.pone.0063181

5. Yagupsky P. Kingella kingae: carriage, transmission, and disease. Clin Microbiol Rev. 2015;28:54-79. https:/ / doi.org/ 10.1128/CMR.00028-14

6. Dubnov-Raz G, Ephros M, Garty BZ, Schlesinger Y, Maayan-Metzger A, Hasson J, et al. Invasive pediatric Kingella kingae infections: a nationwide collaborative study. Pediatr Infect Dis J. 2010;29:639-43. https:/ / doi.org/10.1097/ INF.0b013e3181d57a6c

7. Berbari EF, Cockerill FR III, Steckelberg JM. Infective endocarditis due to unusual or fastidious microorganisms.
Mayo Clin Proc. 1997;72:532-42. https:/ / doi.org/10.4065/ 72.6.532

8. Chambers ST, Murdoch D, Morris A, Holland D, Pappas P, Almela M, et al.; International Collaboration on Endocarditis Prospective Cohort Study Investigators. HACEK infective endocarditis: characteristics and outcomes from a large, multi-national cohort. PLoS One. 2013;8:e63181. https://doi.org/10.1371/journal. pone.0063181

9. Geraci JE, Wilson WR. Symposium on infective endocarditis. III. Endocarditis due to gram-negative bacteria. Report of 56 cases. Mayo Clin Proc. 1982;57:145-8.

10. Thekekara AG, Denham B, Duff DF. Eleven-year review of infective endocarditis. Ir Med J. 1994;87:80-2.

11. Webb R, Voss L, Roberts S, Hornung T, Rumball E, Lennon D. Infective endocarditis in New Zealand children 1994-2012. Pediatr Infect Dis J. 2014;33:437-42. https:/ / doi.org/10.1097/INF.0000000000000133

12. Kelchtermans J, Grossar L, Eyskens B, Cools B, Roggen M, Boshoff D, et al. Clinical characteristics of infective endocarditis in children. Pediatr Infect Dis J. 2019;38:453-8. https:/ / doi.org/10.1097/INF.0000000000002212

13. Amir G, Frenkel G, Rotstein A, Nachum E, Bruckheimer E, Lowenthal A, et al. Urgent surgical treatment of aortic endocarditis in infants and children. Pediatr Cardiol. 2019;40:580-4. https://doi.org/10.1007/s00246-018-2030-5

14. Berkun Y, Brand A, Klar A, Halperin E, Hurvitz H. Kingella kingae endocarditis and sepsis in an infant. Eur J Pediatr. 2004;163:687-8. https:/ / doi.org/10.1007/s00431-004-1520-z

15. Brachlow A, Chatterjee A, Stamato T. Endocarditis due to Kingella kingae: a patient report. Clin Pediatr (Phila). 2004;43:283-6. https:// doi.org/10.1177/000992280404300311

16. Foster MA, Walls T. High rates of complications following Kingella kingae infective endocarditis in children: a case series and review of the literature. Pediatr Infect Dis J. 2014;33:7856. https:// doi.org/10.1097/INF.0000000000000303

17. Martínez Olorón P, Romero Ibarra C, Torroba Álvarez L, Pérez Ocón A. Kingella kingae endocarditis [in Spanish]. An Pediatr (Barc). 2011;74:274-5. https:/ / doi.org/10.1016/ j.anpedi.2010.10.018

18. Rabin RL, Wong P, Noonan JA, Plumley DD. Kingella kingae endocarditis in a child with a prosthetic aortic valve and bifurcation graft. Am J Dis Child. 1983;137:403-4.

19. Rotstein A, Konstantinov IE, Penny DJ. Kingella-infective endocarditis resulting in a perforated aortic root abscess and fistulous connection between the sinus of Valsalva and the left atrium in a child. Cardiol Young. 2010;20:332-3. https://doi.org/10.1017/S1047951110000314

20. Youssef D, Henaine R, Di Filippo S. Subtle bacterial endocarditis due to Kingella kingae in an infant: a case report. Cardiol Young. 2010;20:448-50. https:// doi.org/10.1017/ S1047951110000351

21. Li JS, Sexton DJ, Mick N, Nettles R, Fowler VG Jr, Ryan T, et al. Proposed modifications to the Duke criteria for the diagnosis of infective endocarditis. Clin Infect Dis. 2000;30:633-8. https:// doi.org/10.1086/313753

22. Clinical and Laboratory Standards Institute. Performance standards for antimicrobial susceptibility testing: 25th edition informational supplement (M100-S25). Wayne (PA): The Institute; 2015.

23. Yoshida K, Bohn J. tableone-package: create "Table 1" to describe baseline characteristics [cited 2020 Jul 26]. https://rdrr.io/cran/tableone/man/tableone-package.html

24. Marom D, Birk E, Ashkenazi S. Trends in pediatric infective endocarditis in a tertiary pediatric center in Israel [in Hebrew]. Harefuah. 2012;151:464-8, 98, 97. 
25. Gupta S, Sakhuja A, McGrath E, Asmar B. Trends, microbiology, and outcomes of infective endocarditis in children during 2000-2010 in the United States. Congenit Heart Dis. 2017;12:196-201. https:/ / doi.org/10.1111/ chd.12425

26. Baddour LM, Wilson WR, Bayer AS, Fowler VG Jr, Tleyjeh IM, Rybak MJ, et al.; American Heart Association Committee on Rheumatic Fever, Endocarditis, and Kawasaki Disease of the Council on Cardiovascular Disease in the Young, Council on Clinical Cardiology, Council on Cardiovascular Surgery and Anesthesia, and Stroke Council. Infective endocarditis in adults: diagnosis, antimicrobial therapy, and management of complications: a scientific statement for healthcare professionals from the American Heart Association. Circulation. 2015;132:1435-86. https://doi.org/10.1161/CIR.0000000000000296

27. Amit U, Porat N, Basmaci R, Bidet P, Bonacorsi S, Dagan R, et al. Genotyping of invasive Kingella kingae isolates reveals predominant clones and association with specific clinical syndromes. Clin Infect Dis. 2012;55:1074-9. https:/ / doi.org/ 10.1093/cid/cis622

28. Bárcena-Uribarri I, Benz R, Winterhalter M, Zakharian E, Balashova N. Pore forming activity of the potent RTX-toxin produced by pediatric pathogen Kingella kingae:

Characterization and comparison to other RTX-family members. Biochim Biophys Acta. 2015;1848:1536-44. https://doi.org/10.1016/j.bbamem.2015.03.036

29. Hoerr V, Franz M, Pletz MW, Diab M, Niemann S, Faber C, et al. S. aureus endocarditis: Clinical aspects and experimental approaches. Int J Med Microbiol. 2018;308:640-52. https://doi.org/10.1016/j.ijmm.2018.02.004

Address for correspondence: Oded Scheuerman, Schneider Children's Medical Center of Israel, 14 Kaplan St, PO Box 559, Petach Tikva 49202, Israel; email: odedshv@clalit.org.il

\section{The Public Health Image Library (PHIL)}
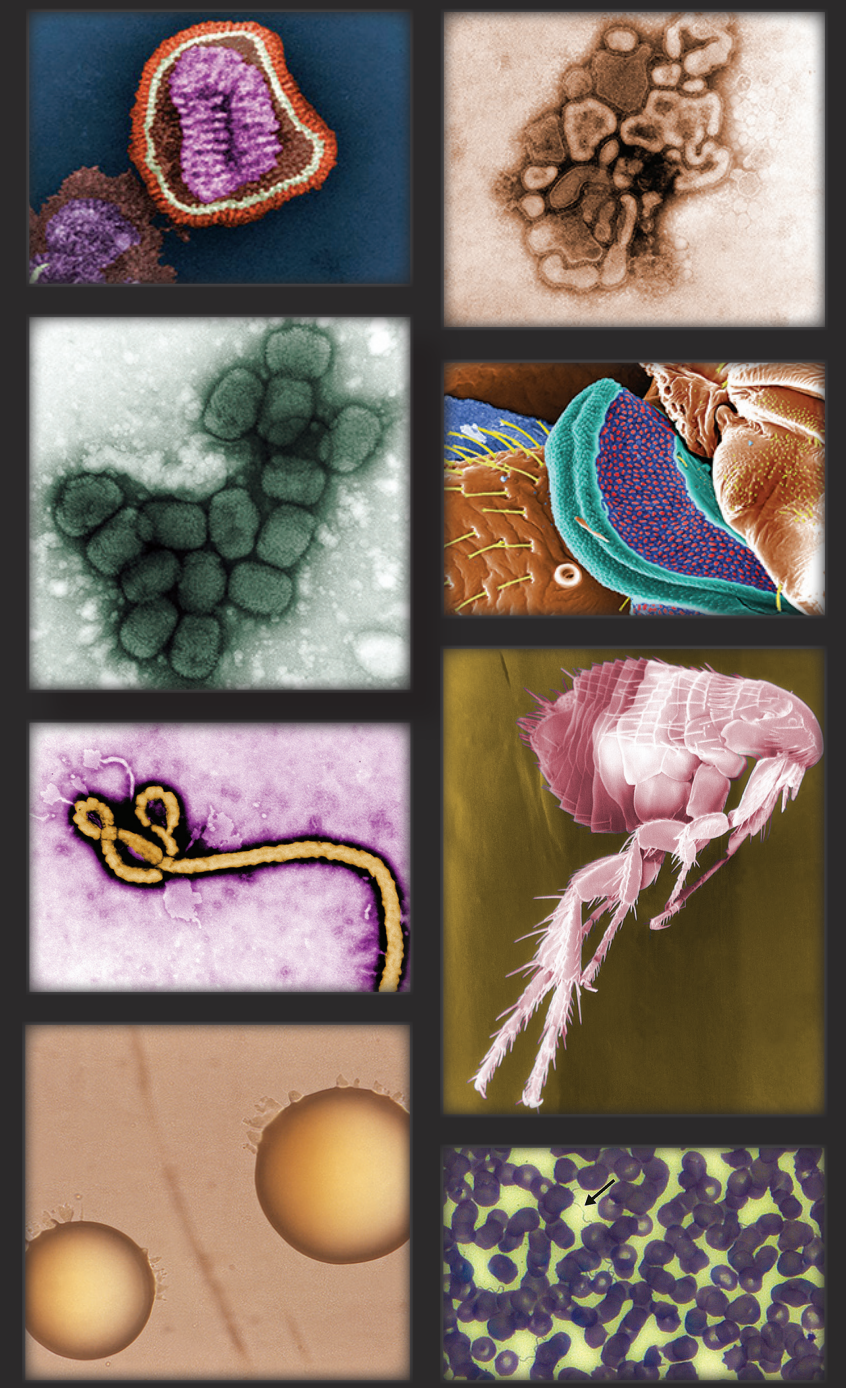

The Public Health Image Library (PHIL), Centers for Disease Control and Prevention, contains thousands of public healthrelated images, including high-resolution (print quality) photographs, illustrations, and videos.

PHIL collections illustrate current events and articles, supply visual content for health promotion brochures, document the effects of disease, and enhance instructional media.

PHIL images, accessible to PC and Macintosh users, are in the public domain and available without charge.

Visit PHIL at: http: / / phil.cdc.gov/phil 